\title{
Quantitative Analysis of Three-dimensional Fatigue Fracture Surface Reconstructed by Stereo Matching Method
}

\author{
Manabu TANAKA, Yosuke KIMURA, ${ }^{1)}$ Lotfi CHOUANINE, Junnosuke TAGUCHI, ${ }^{2)}$ and Ryuichi KATO \\ Department of Mechanical Engineering, Faculty of Engineering and Resource Science, Akita University, Tegatagakuen-cho, \\ Akita 010-8502 Japan. $\quad$ 1) Nippon Systemware Company, Sakuragaoka-machi, Shibuya-ku, Tokyo $150-8577$ Japan. \\ 2) Graduate Student, Department of Mechanical Engineering, Faculty of Engineering and Resource Science, Akita University, \\ Tegatagakuen-cho, Akita 010-8502 Japan.
}

(Received on December 4, 2002; accepted in final form on April 4, 2003)

\begin{abstract}
Three-dimensional geometry of a fatigue fracture surface in a $\mathrm{Cu}-\mathrm{Be}$ alloy (the grain diameter is about $24 \mu \mathrm{m}$ ) was reconstructed by the stereo matching method based on the coarse-to-fine method. The fractal dimensions of the contours and of the fracture surface profiles extracted from the reconstructed image were then estimated by the box-counting method in the scale length range smaller than about one grainboundary length (about $14 \mu \mathrm{m}$ ). The mean value of the fractal dimension of the contours was about 1.238, and was close to that of the actual fracture surface profiles in the plane in parallel with the crack growth direction (about 1.210) (in the parallel direction) and in the plane perpendicular to the crack growth direction (about 1.190) (in the perpendicular direction). Thus, the stereo matching method can reproduce the three-dimensional image of the complex fracture surface. However, the mean value of the fractal dimension of the reconstructed fracture surface profiles (about 1.128 in the parallel direction and about 1.146 in the perpendicular direction) was a little smaller than that of the actual fracture surface profiles, since there were microstructural features such as overhang, debris and microcracks on the fatigue fracture surface, which were only partly reproducible by the stereo matching method. Nevertheless, the geometrical information about a given fracture surface in a wide area can be obtained by the three-dimensional fracture surface reconstruction and analysis. Combination of three-dimensional and two-dimensional analyses may lead to the further understanding of the geometrical features of fracture surfaces and the fracture mechanism in materials. The fractal dimension was found to be a useful index not only for characterizing fracture surfaces but also for comparison of the result of three-dimensional image reconstruction with the actual fracture surface morphology.
\end{abstract}

KEY WORDS: stereo matching method; three-dimensional geometry; coarse-to-fine method; fractal dimension; fracture surface; three-dimensional image reconstruction; $\mathrm{Cu}-\mathrm{Be}$ alloy.

\section{Introduction}

Computer-aided stereo matching method using a stereo pair (i.e., basic image and tilted image) has been successfully applied to the reconstruction of three-dimensional images. ${ }^{1-3)}$ The procedure of three-dimensional image reconstruction is principally composed of the detection of corresponding points in a stereo pair by the pattern recognition, and the calculation of height at all the corresponding points. ${ }^{1-4)}$ Stereo matching method has also been applied to the three-dimensional image reconstruction of fracture surfaces in materials. ${ }^{1,2,4-8)}$ Kobayashi and Shockey ${ }^{5)}$ and Stampfl et al. $\left.{ }^{6}\right)$ estimated the fracture toughness of steels from the reconstructed three-dimensional images. Kimura and Tanaka. ${ }^{8}$ proposed a new stereo matching method on the basis of the coarse-to-fine method for facilitating the three-dimensional image reconstruction of fracture surfaces. However, a quantitative analysis has not sufficiently been made for describing the geometrical features of reconstructed three-dimensional fracture surfaces in materials.
Mandelbrot et al. ${ }^{9)}$ first revealed that in impact-loaded and fractured steels fracture surfaces have a self-similarity and can be characterized by the fractal dimension. Since then, fractal analysis has been applied to the quantitative description of fracture surfaces ${ }^{10-15)}$ and microstructures $^{16-19)}$ in materials. The fractal dimension represents the extent of geometrical complexity in self-similar objects in statistical sense, although a certain value of the fractal dimension does not uniquely correspond to a specific pattern of fracture surfaces or microstructures. The scale length range, in which the fractal dimension is estimated, is associated with the size range of characteristic microstructures or fracture patterns. ${ }^{10,15,17)}$ Thus, the fractal dimension seems to be a useful index in order to examine the geometrical similarity between patterns of different objects. The surface roughness (the root mean square) of the fracture surface profiles was also examined on the actual fracture surfaces and on the reconstructed image for comparison purpose.

In this study, a three-dimensional image was reconstructed by the stereo matching method proposed by Kimura and 
Tanaka on the complex fatigue fracture surface of a $\mathrm{Cu}-\mathrm{Be}$ alloy (the grain diameter is about $24 \mu \mathrm{m}$ ). ${ }^{8)}$ The fractal dimension was estimated by the box-counting method ${ }^{20,21)}$ on the fracture surface profiles in the plane in parallel with the crack growth direction (shortly, in the parallel direction) or in the plane perpendicular to the crack growth direction (in the perpendicular direction) as well as the contours, which were extracted from the reconstructed image. In connection with the size ranges of the related microstructures, the fractal dimension was estimated in the scale length range smaller than about one grain-boundary length (about $14 \mu \mathrm{m}) .{ }^{15)}$ Reproducibility of the three-dimensional image reconstruction by the stereo matching method was then examined by comparing the results of the fractal analysis on the reconstructed image with those obtained on the actual fracture surface profiles. ${ }^{15)}$

\section{Stereo Matching Method}

\subsection{Template Matching}

The information in the height direction can be obtained by the stereo matching method using a stereo pair (the basic image and the tilted image). The $x$-coordinate of a point $\mathrm{P}$ on the fracture surface, $x$ (on the basic image) moves to that of the corresponding point $\mathrm{P}^{\prime}, x^{\prime}$ (on the tilted image) in the $x$-direction on the $x-y$ plane (on the base plane) by tilting a specimen around the $y$-axis $(\theta)$, as shown in Fig. 1. The reference plane of the basic image is the $x-y$ plane and that of the tilted image is the $x^{\prime}-y$ plane in this figure. The height of the point $\mathrm{P}$ from the base plane, $\mathrm{h}$, can be obtained by searching the corresponding point (homologous point) $\mathrm{P}^{\prime}$ in the tilted image. Since the height of these points from each reference plane is the same (Fig. 1), the value of $h$ is given by the following equation with the magnitude of image $M$ :

$$
h=\frac{1}{M}\left(\frac{x}{\tan \theta}-\frac{x^{\prime}}{\sin \theta}\right)
$$

It is necessary for reconstructing a three-dimensional image from a stereo pair to examine the pixel-to-pixel correspondence between the basic image and the tilted image. ${ }^{1-4)}$ The template matching was employed to detect the corresponding point (pixel) in these two images. The window of a given size ( $q$ pixels $\times r$ pixels) in the basic image was imposed on that of the same size in the searched area ( $k$ pixels $\times l$ pixels) of the tilted image in order to examine the similarity of these windows, as shown in Fig. 2. In this study, the correlation factor between these windows was calculated using the color number (the brightness) of each pixel. The homologous (corresponding) point was identified when the correlation factor, $R_{\mathrm{C}}$, given by the following equation has the maximum value in the searched $\operatorname{area}^{2,7,22)}$.

$$
R_{\mathrm{C}}=\frac{\sum_{i j}\left(T_{i j} \cdot S_{i j}\right)-N \cdot \bar{T} \cdot \bar{S}}{\sqrt{\left(\sum_{i j} T_{i j}^{2}-N \cdot \bar{T}^{2}\right)\left(\sum_{i j} S_{i j}^{2}-N \cdot \bar{S}^{2}\right)}}
$$

where $N$ is the number of pixels in a template, and $T_{i j}$ and

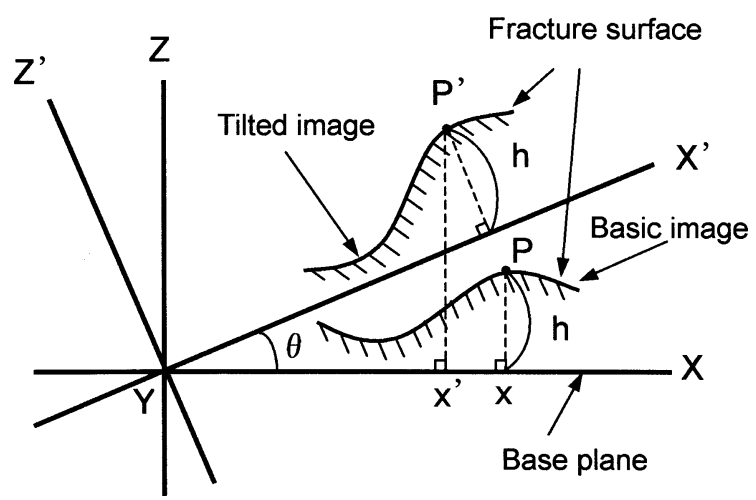

Fig. 1. Displacement of the $x$-coordinate of a point $\mathrm{P}$ from $x$ (on the basic image) to that of the corresponding point $\mathrm{P}^{\prime}, x^{\prime}$ (on the tilted image) in the $x$-direction on the $x-y$ plane (on the base plane) by tilting a specimen around the $y$ axis $(\theta)$ ( $h$ is the height of the point $\mathrm{P}$ from the reference plane).

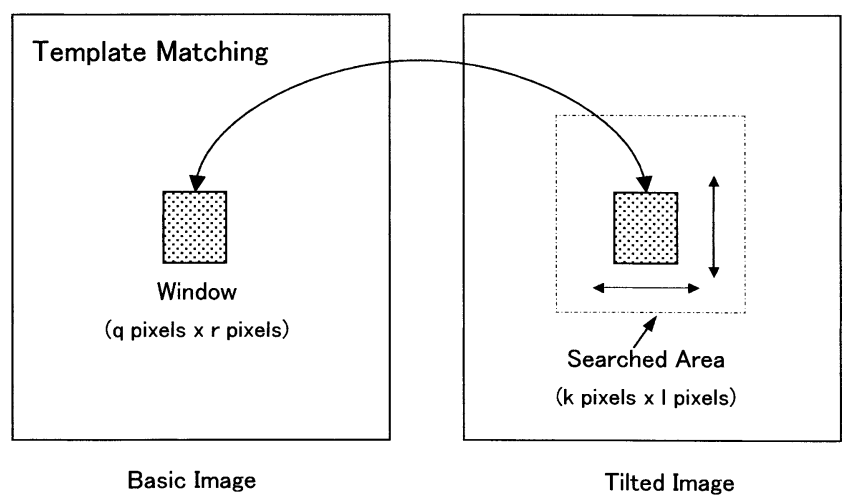

Fig. 2. Schematic illustration of the template matching between the basic image and the tilted image.

$S_{i j}$ are the brightness (color number) of a pixel located at ( $i$, $j$ ) in the template for the window in the basic image and for the imposed area in the tilted image, respectively. The averaged values of the brightness are $\bar{T}=(1 / N) \sum_{i j} T_{i j}$ and $\bar{S}=(1 / N) \sum_{i j} S_{i j}$.

\subsection{Stereo Matching Method Based on Coarse-to-Fine Method}

In the template matching, the template size should be large enough for obtaining reasonable results of the matching ${ }^{1,4,23)}$, although the amount of calculation increases with increasing the template (window) size or with increasing the searched area. Therefore, it is necessary to reduce time for template matching, especially on stereo pair with the image size of more than some hundred thousands pixels. In this study, the coarse-to-fine method was employed for facilitating the template matching. Figure 3 shows the procedure of three-dimensional image reconstruction by the coarse-to-fine method. The images with the reduced resolution by $1 / 2,1 / 4$, and $1 / 8$ were successively produced by "down sampling" (Down Sampling 1 to 3 in the figure) using the original images (the basic image and the tilted image) in this study. The template matching for three-dimensional image reconstruction was then carried out from the image with the lowest resolution up to that with the highest resolution (the original image) in order to detect the corresponding points between the basic image and the tilted 
image. This procedure is also shown by the numbers (1) to (8) in the figure. In the matching process, two kinds of the spatial filter, namely, the median value filter and the mean

(7)'

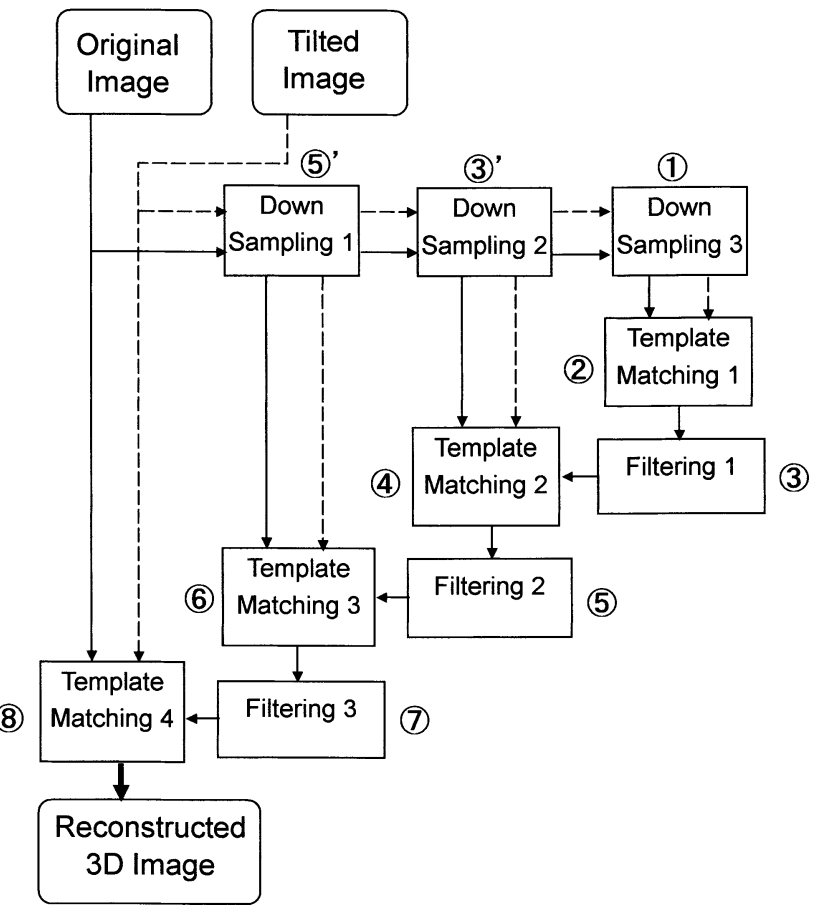

Fig. 3. The procedure of the three-dimensional (3D) image reconstruction by the stereo matching method on the basis of the coarse-to-fine method in this study (the template matching and the filtering were made from the image with the lowest resolution obtained after Down Sampling 3 to the original image (Template Matching 4), as indicated by the numbers from (1) to (8)).

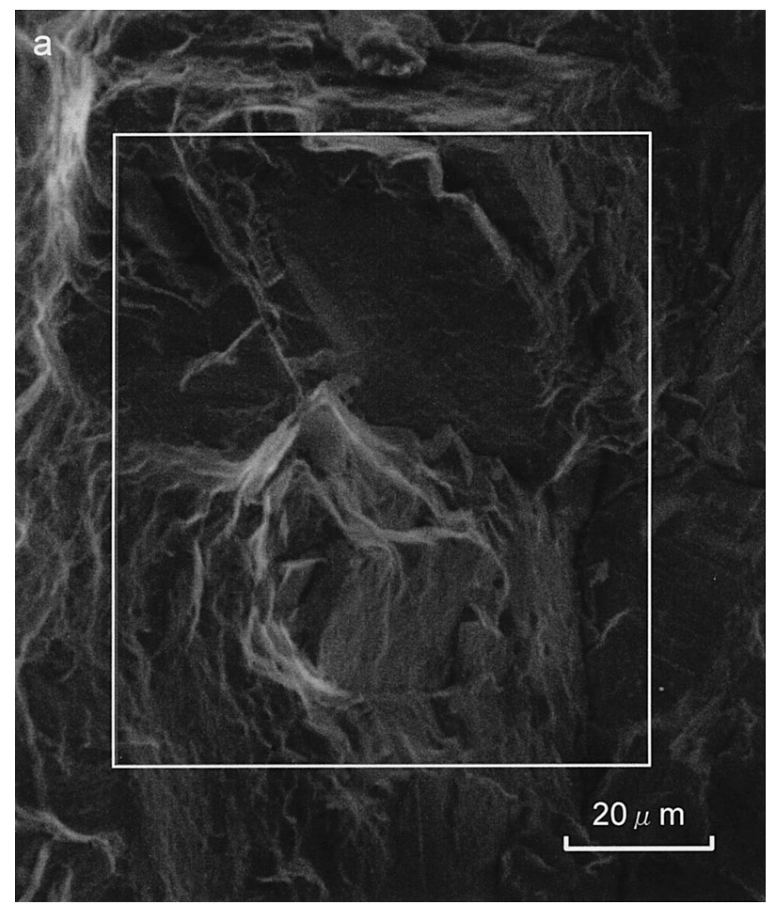

value filter were also used at each step of template matching to eliminate or to reduce the effects of mismatching and noise on the reconstruction of three-dimensional images. The coarse-to-fine method enables fast template matching with a template (window) of small size in small searched area.

A computer program, which was made by Visual Basic version 6.0, was developed and used for the reconstruction, processing and analysis of three-dimensional images in this study. Another computer program used in the previous study was also employed for the fractal analysis of the processed images by the box-counting method ${ }^{20,21,24,25)}$.

\section{Results}

\subsection{Reconstruction of Three-dimensional Fracture Surface}

A fatigue fracture surface of a $\mathrm{Cu}-\mathrm{Be}$ alloy (the mean grain diameter is about $24 \mu \mathrm{m}$ ) was obtained by repeated bending of a specimen with fatigue testing equipment at the maximum total strain range $\Delta \varepsilon_{\mathrm{t}}=0.0171$ on the specimen surface (the mean fatigue life over six specimens was 3567 cycles ${ }^{15)}$. Photographs of stereo pair (the basic image and the tilted image with a tilting angle of $10^{\circ}$ ) were then taken using a scanning electron microscope (SEM) on the fatigue fracture surface at the location of about $0.2 \mathrm{~mm}$ inside from the specimen surface (the region of stage I fatigue crack growth) at the magnification of 700 times. Photographs were taken into a personal computer using an image scanner as digital images in 256 grey scale levels with the resolution of $200 \mathrm{dpi}$.

Figure 4 shows the computed region for the three-dimensional image reconstruction and the brightness (height)

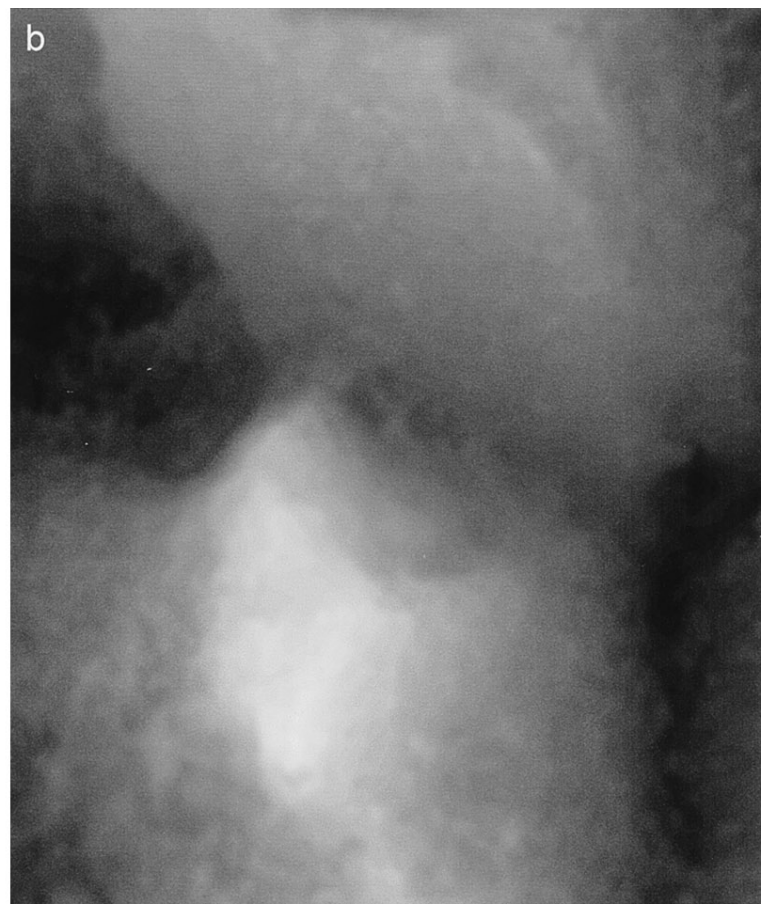

Fig. 4. Computed region for the three-dimensional image reconstruction and the brightness (height) image generated from the reconstructed three-dimensional image of the fatigue fracture surface in a $\mathrm{Cu}-\mathrm{Be}$ alloy. (a) Computed region on the basic image (the region enclosed by white line is 338 pixels $\times 402$ pixels). (b) Brightness (height) image (the template size for image reconstruction is 5 pixels $\times 5$ pixels). 
image generated from the reconstructed three-dimensional image. The macroscopic direction of fatigue crack growth is approximately from right to left in Fig. 4(a). The fracture surface is composed of slip steps in the grains and grainboundary facets with dimples, which were nucleated at grain-boundary precipitates ${ }^{15}$. Thus, the fatigue fracture surface is considerably complicated with these microstructural features. In Fig. 4(a) is also shown the computed region on the basic image for the reconstruction of the threedimensional image of the fatigue fracture surface (the region is enclosed by white line). The stereo matching for the three-dimensional image reconstruction was carried out using the correlation factor for detection of corresponding points in stereo pair with the template (window) size of 5 pixels $\times 5$ pixels, since the optimum results of the stereo matching method were obtained under this condition ${ }^{8)}$. Figure 4(b) shows the brightness (height) image of the reconstructed three-dimensional fatigue fracture surface. The relative height on the fracture surface is shown by the brightness (color number) in the image. The relative height increases with increasing the color number (brightness), and the brightest part in the image indicates the highest part on the fracture surface. Fine geometrical features of the fracture surface are reproduced in the reconstructed image. The resolution of the reconstructed image was about $0.17 \mu \mathrm{m}$ in the plane in parallel with the fracture surface.

Figure 5 shows the bird's-eye view of the reconstructed three-dimensional fatigue fracture surface in a $\mathrm{Cu}-\mathrm{Be}$ alloy. Complex three-dimensional geometry of the fatigue fracture surface can be reproduced by the stereo matching method, although the figure shows only principal features of the fracture surface. Crack growth direction is approximately from lower right to upper left in the figure.

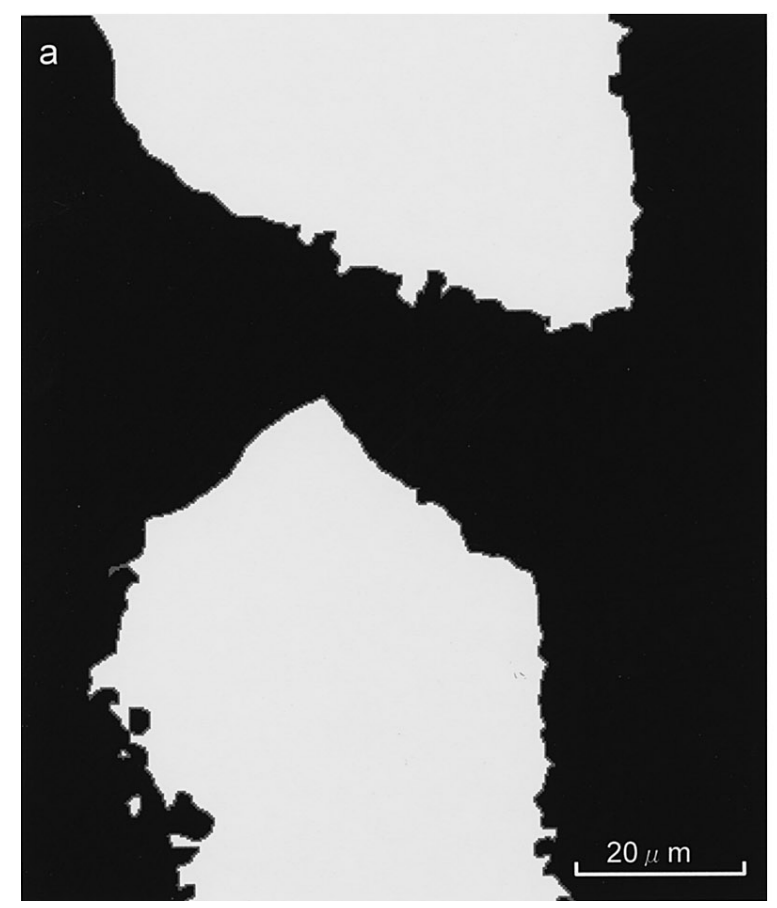

\subsection{Fractal Analysis of the Reconstructed Image}

The reconstructed three-dimensional image of the fatigue fracture surface was level-sliced and was then processed for fractal analysis of the contours. Figure 6 shows an example of processed binary images of the cross section and the contours used for fractal analysis in the reconstructed threedimensional fracture surface. In Fig. 6(b), the lines with one pixel width indicating the contours were drawn by using a software between the white region (material) and the black region. Fig. 6(a) corresponds to the No. 8 contour in Fig. 6(b). The fractal dimension of the contours superimposed on the height (brightness) image was estimated on representative contours shown in Fig. 6(b). The numbers in the figure indicate the relative height. Figure 7 shows the

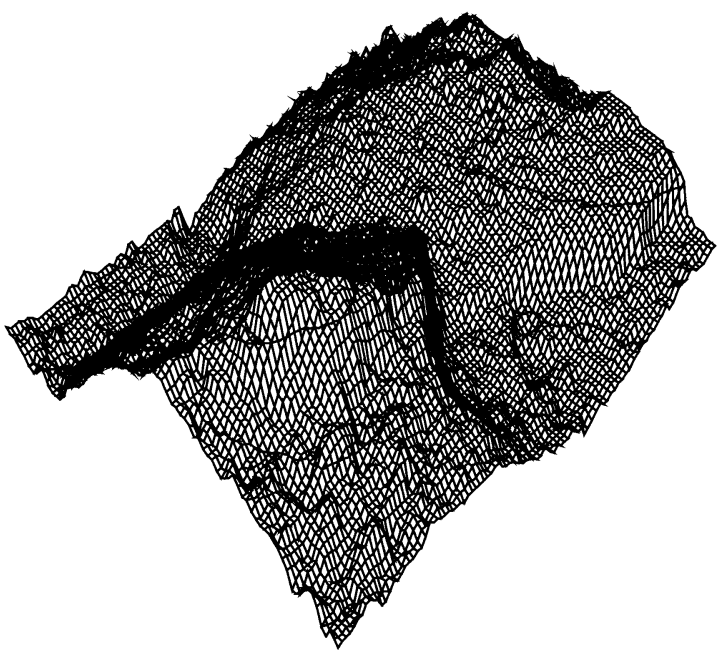

Fig. 5. Bird's-eye view of the reconstructed three-dimensional image of the fatigue fracture surface in a $\mathrm{Cu}-\mathrm{Be}$ alloy (the datum points are 85 points $\times 101$ points).

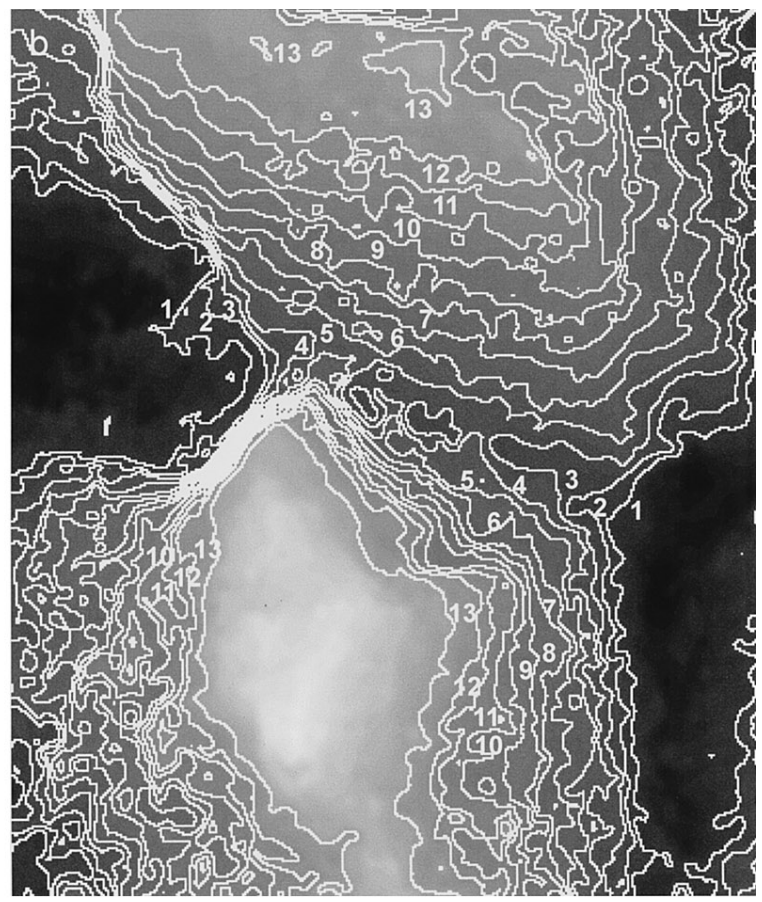

Fig. 6. An example of processed binary images of the cross section and the contours used for the fractal analysis in the reconstructed three-dimensional fracture surface. (a) Processed binary image (No. 8 contour in b). (b) Contours used for fractal analysis (numbers indicate the relative height). 
fractal dimension of thirteen contours (Fig. 6(b)) in the reconstructed three-dimensional image. The fractal dimension of the contours was estimated in the scale length range smaller than about one grain-boundary length (about $14 \mu \mathrm{m})$. The fractal dimension lies between about 1.17 and about 1.29, and the mean value of the fractal dimension is about 1.238. Also shown in the figure is the fractal dimension of the actual fracture surface profiles in two directions. The mean value of the fractal dimension of the actual fracture surface profiles was about 1.210 in the plane in parallel with the crack growth direction (parallel direction) ${ }^{15)}$ and about 1.190 in the plane perpendicular to the crack growth direction (perpendicular direction). The fractal dimension of the contours obtained in this study is close to that of the fracture surface profiles. Thus, the stereo matching method can reproduce the three-dimensional image of the complex fracture surface. The fatigue fracture surface in the $\mathrm{Cu}-\mathrm{Be}$

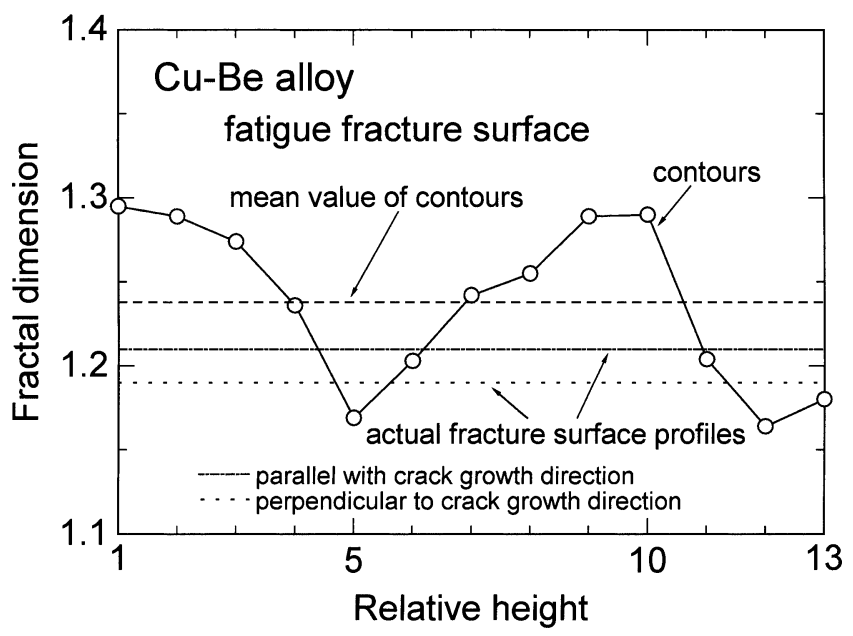

Fig. 7. The fractal direction of thirteen contours (Fig. 6(b)) extracted from the reconstructed three-dimensional image of the fatigue fracture surface in a $\mathrm{Cu}-\mathrm{Be}$ alloy.

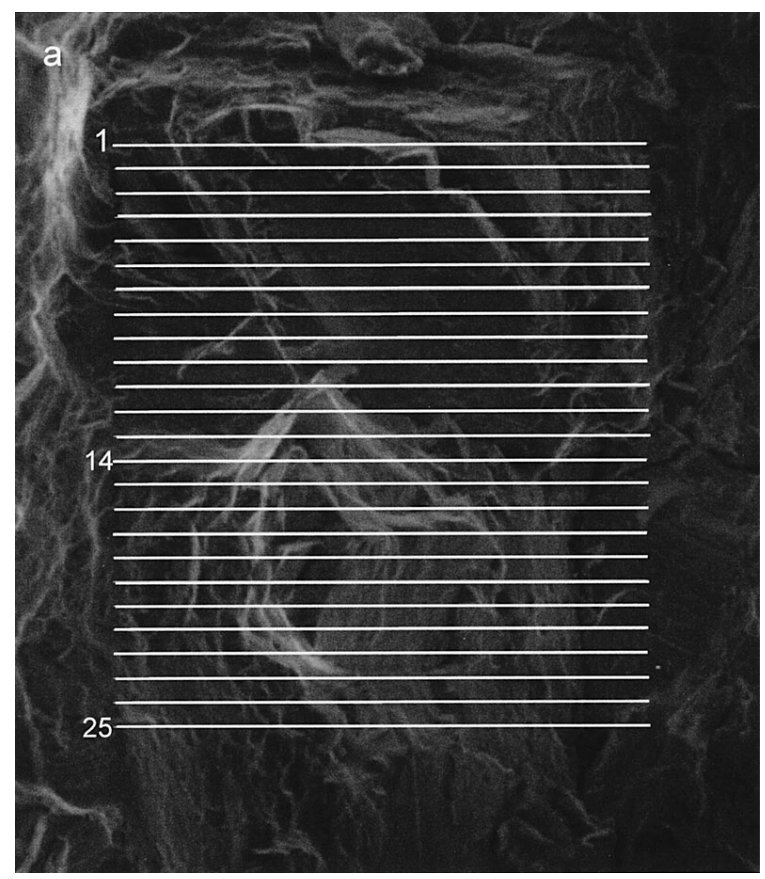

alloy seems to be isotropic at least in the length scale range smaller than about one grain-boundary length.

Figure 8 shows the sampling locations of the fatigue fracture surface profiles in the reconstructed three-dimensional image. The fractal dimension of the fracture surface profiles was examined by the box-counting method in both the parallel (Fig. 8(a)) and perpendicular directions (Fig. $8(b))$ in the same way as the fractal analysis of the contours. Figure 9 shows the fractal dimension of the fatigue fracture surface profiles in the parallel direction. The fractal dimension changes from about 1.08 to about 1.22 with the sampling location of profiles. As shown in the figure, the mean value of the fractal dimension is about 1.128 and is smaller than that of the actual fracture surface profiles (1.210) in the parallel direction. A similar tendency can be observed on the fractal dimension of the fracture surface profiles in the perpendicular direction (Fig. 10). The fractal dimension is in the range between about 1.09 and about 1.19 in this

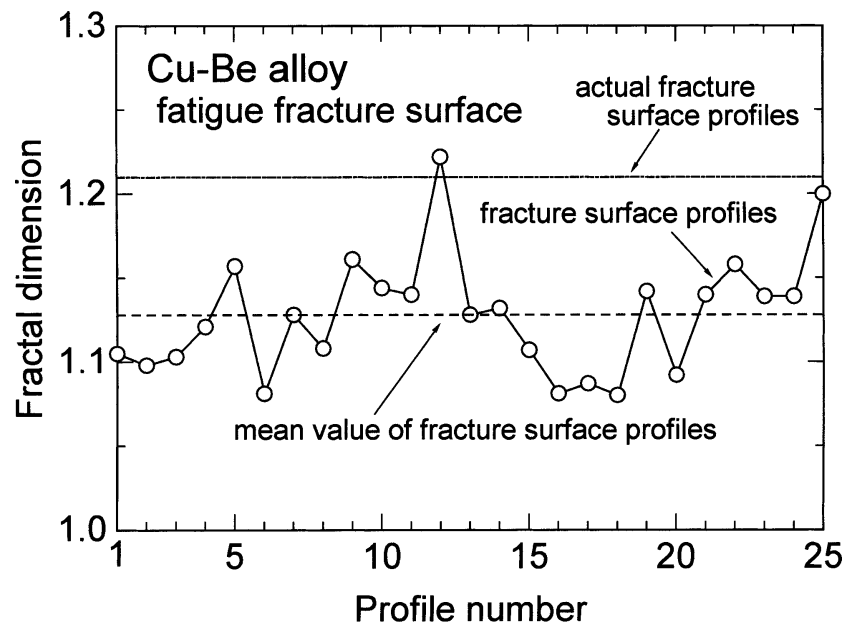

Fig. 9. The fractal dimension of the fatigue fracture surface profiles in the parallel direction.

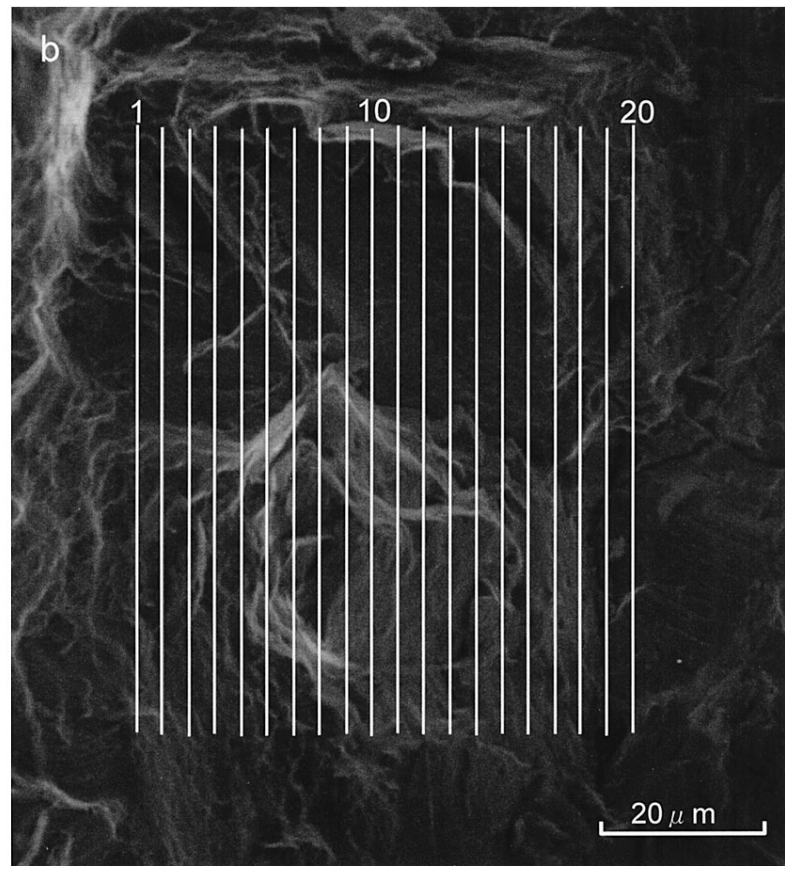

Fig. 8. Sampling locations for fractal analysis of the fatigue fracture surface profiles extracted from the reconstructed three-dimensional image. (a) Profiles in the plane in parallel with the crack growth direction (in the parallel direction). (b) Profils in the plane perpendicular to the crack growth direction (in the perpendicular direction). 
case. The mean value of the fractal dimension is about 1.146 and is a little smaller than that of the actual fracture surface profiles (1.190) in the perpendicular direction.

Figure 11 shows examples of the fracture surface profiles in both parallel and perpendicular directions extracted from the reconstructed three-dimensional image. The profile number in the figure corresponds to that shown in Fig. 8 . The fractal dimension of these fracture surface profiles (1.132 in the parallel direction and 1.156 in the perpendicular direction) is a little smaller than that of the actual fracture surface profiles (1.210 in the parallel direction and 1.190 in the perpendicular direction). As shown in the figure caption, the larger value of the surface roughness (the root mean square, rms) does not correspond to the larger fractal dimension in this case. The fracture surface profiles extracted from the reconstructed image seem to be a little simpler in detail than the actual fracture surface profiles in the fatigued specimens of a $\mathrm{Cu}-\mathrm{Be}$ alloy, while the reconstructed image reproduces principal features of the actual fracture surface.

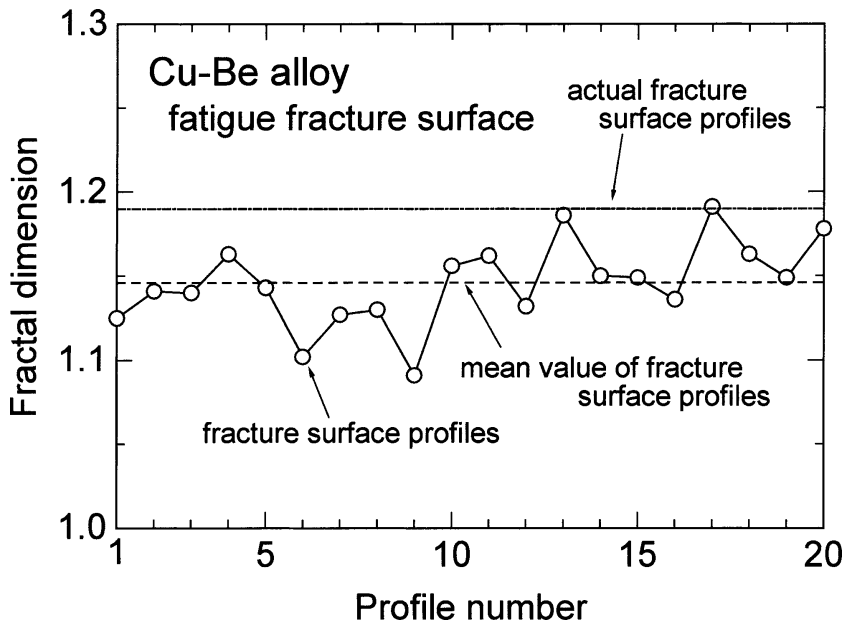

Fig. 10. The fractal dimension of the fatigue fracture surface profiles in the perpendicular direction.

\section{Discussion}

\subsection{Reconstructed and Actual Fracture Surface Pro- files}

Figure 12 shows examples of the actual fatigue fracture surface profiles in the fatigued specimen of a $\mathrm{Cu}-\mathrm{Be}$ alloy. These micrographs were taken on different specimens used in the previous study ${ }^{15)}$. The fractal dimension of the fracture surface profiles is 1.197 in the parallel direction (Fig. 12(a)) and 1.180 in the perpendicular direction (Fig. 12(b)). Similar values of the fractal dimension (i.e. similar extents of the geometrical complexity) of the fracture surface pro-
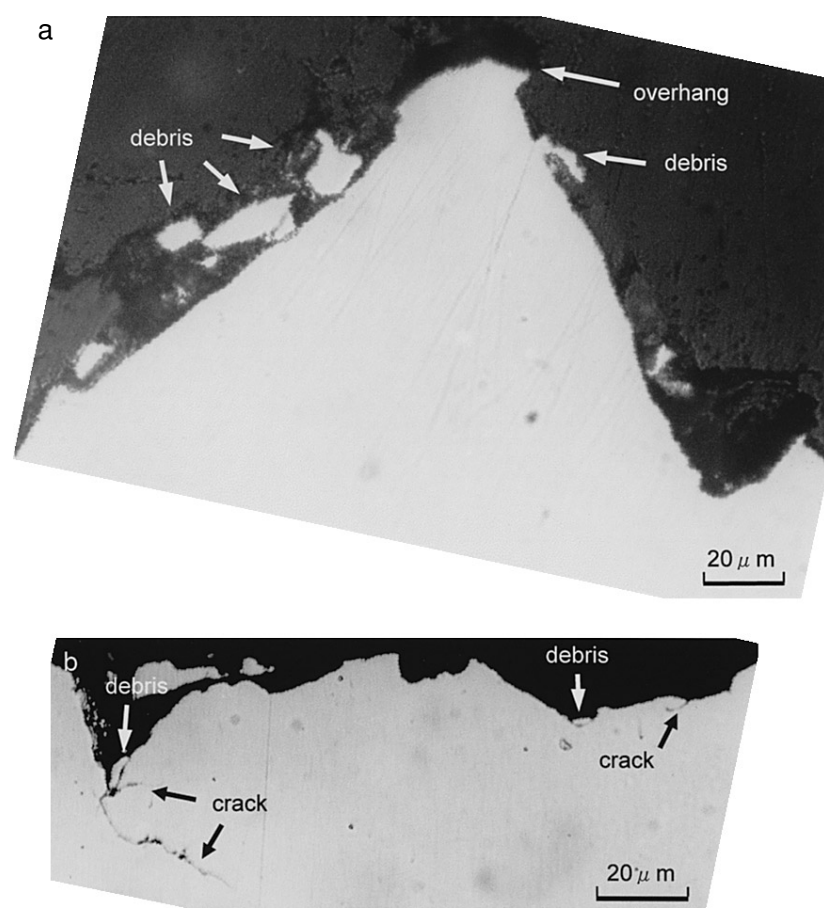

Fig. 12. Examples of fracture surface profiles in the fatigued specimen of a $\mathrm{Cu}-\mathrm{Be}$ alloy. (a) A profile in the parallel direction $(D=1.197, \mathrm{rms}=36.3 \mu \mathrm{m})$. (b) A profile in the perpendicular direction $(D=1.180, \mathrm{rms}=5.67 \mu \mathrm{m})$. $(D$ : the fractal dimension of the fracture surface profile; rms: the surface roughness (root mean square).)
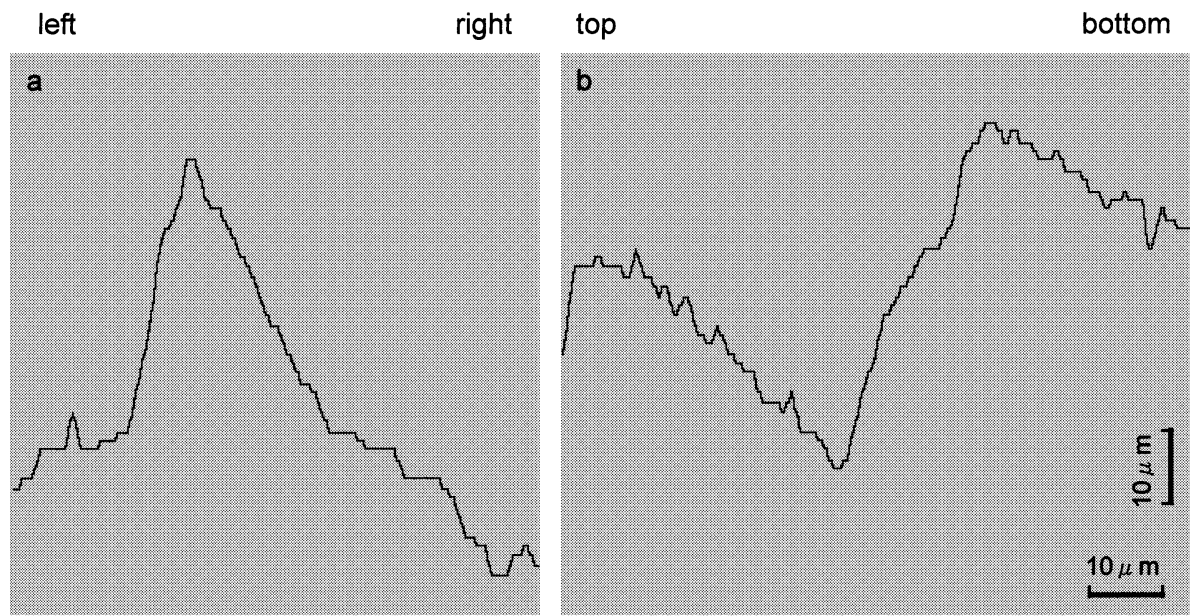

Fig. 11. Examples of fracture surface profiles extracted from the reconstructed three-dimensional image of the fatigue fracture surface in a $\mathrm{Cu}-\mathrm{Be}$ alloy. (a) No. 14 profile in the parallel direction $(D=1.132, \mathrm{rms}=11.9 \mu \mathrm{m})$. (b) No 10 profile in the perpendicular direction $(D=1.156, \mathrm{rms}=10.1 \mu \mathrm{m})$. ( $(D$ : the fractal dimension of the fracture surface profile; rms: the surface roughness (root mean square).) 
files suggest that these fracture surfaces were formed by a common mechanism, namely, a mixed mode of slipping-off in the grains and grain-boundary fracture with dimples ${ }^{15}$, whereas the surface roughness (rms) is very different in these two specimens. As shown by arrows, there are overhang (Fig. 12(a)) and debris (Figs. 12(a) and 12(b)) on the fracture surface profile. There are also microcracks linked to the fracture surface (Fig. 12(b)). These are considered to be microstructural features of the fracture surface ${ }^{26)}$, which are only partly reproducible by the stereo matching method and may not fully be detected by a scanning tunneling microscope (STM) or an atomic force microscope (AFM). This is why a smaller value of the fractal dimension was obtained on the fracture surface profiles extracted from the reconstructed three-dimensional image (Figs. 9, 10 and 11).

Kobayashi and Shockey ${ }^{5}$ reported that the fracture toughness for crack initiation $\left(J_{\mathrm{IC}}\right)$ estimated from the reconstructed fracture surface profiles coincided with the value obtained using a conventional fracture test procedure in steels. Stampfl and Kolednik ${ }^{7)}$ correlated the local fracture toughness estimated from the reconstructed images with the specific microstructures. Their results also indicate that the three-dimensional image reconstruction using the stereo matching method can reproduce the principal features of fracture surfaces and is a useful tool for the estimation of fracture toughness values or for the study of micro fracture mechanisms in materials. Two-dimensional analysis of actual fracture surface profiles can reveal microstructures such as debris, overhang and microcracks linked to the fracture surface, which cannot be detected by three-dimensional fracture surface analysis ${ }^{10,11,14,15,27)}$. However, the geometrical information about a given fracture surface in a wide area can be obtained by the three-dimensional fracture surface analysis ${ }^{1,2,4-8)}$. Combination of three-dimensional and two-dimensional analyses may lead to the further understanding of the geometrical features of fracture surfaces and the fracture mechanism in materials.

\subsection{Fractal Dimension and Surface Roughness}

The surface roughness depended on the profile length and tended to increase with increasing profile length. Further, as shown in Fig. 12, different fracture surface profiles often have very different values of the surface roughness $(36.3 \mu \mathrm{m}$ and $5.67 \mu \mathrm{m})$, even if they have the geometrical similarity (the fractal dimension of the fracture surface profiles are 1.197 and 1.180). Therefore, the surface roughness (rms) does not seem to be a suitable parameter to describe the morphological features of fracture surfaces. On the contrary, the fractal dimension of the fracture surface profiles can characterize the geometrical features of the fatigue fracture surface in the length scale range smaller than one grain-boundary length, which is associated with the size range of characteristic microstructures such as slip steps and dimple patterns on the fracture surface ${ }^{10,15)}$. Thus, the fractal dimension of the contours and that of the fracture surface profiles are considered to be a useful index not only for characterizing fracture surfaces but also for comparison of the result of three-dimensional image reconstruction with the actual fracture surface morphology.

\section{Conclusions}

Three-dimensional geometry of a fatigue fracture surface in a $\mathrm{Cu}-\mathrm{Be}$ alloy was reconstructed by the stereo matching method based on the coarse-to-fine method. The fractal dimensions of the contours and of the fracture surface profiles extracted from the reconstructed image were then estimated by the box-counting method in the scale length range smaller than about one grain-boundary length (about $14 \mu \mathrm{m})$. The results of the fractal analysis were compared with those obtained on the actual fracture surface profiles. The results obtained were summarized as follows.

(1) The mean value of the fractal dimension of the contours was about 1.238 , and was close to the values of the actual fracture surface profiles in the plane in parallel with the crack growth direction (about 1.210) and in the plane perpendicular to the crack growth direction (about 1.190). Thus, the stereo matching method can reproduce the threedimensional image of the complex fracture surface. The fatigue fracture surface in the $\mathrm{Cu}-\mathrm{Be}$ alloy seems to be isotropic at least in the length scale range smaller than about one grain-boundary length.

(2) The mean value of the fractal dimension of the reconstructed fracture surface profiles (about 1.128 in the plane in parallel with the fatigue crack growth direction and about 1.146 in the plane perpendicular to the crack growth direction) was a little smaller than that of the actual fracture surface profiles. There were microstructural features such as overhang, debris and microcracks on the fatigue fracture surface of a $\mathrm{Cu}-\mathrm{Be}$ alloy, which were only partly reproducible by the stereo matching method. This was why a smaller value of the fractal dimension was obtained on the fracture surface profiles extracted from the reconstructed three-dimensional image.

(3) The geometrical information about a given fracture surface in a wide area can be obtained by the three-dimensional fracture surface reconstruction and analysis. Combination of three-dimensional and two-dimensional analyses may lead to the further understanding of the geometrical features of fracture surfaces and the fracture mechanism in materials.

(4) The fractal dimension of the fracture surface profiles can characterize the geometrical features of the fatigue fracture surface in the length scale range smaller than one grain-boundary length, which is associated with the size range of characteristic microstructures such as slip steps and dimple patterns on the fracture surface. The fractal dimension is a useful index not only for characterizing fracture surfaces but also for comparison of the result of threedimensional image reconstruction with the actual fracture surface morphology.

(5) The surface roughness depended on the profile length and tended to increase with increasing profile length. Different fracture surface profiles often have very different values of the surface roughness, even if they have the geometrical similarity. Thus, the surface roughness is not a suitable parameter to describe the morphological features of fracture surfaces. 


\section{Acknowledgements}

The authors thank The Iron and Steel Institute of Japan (Tekkou-Kenkyu-Josei[b]) and Mitsutoyo Association for Science and Technology (MAST) for financial support.

\section{REFERENCES}

1) K. Komai and J. Kikuchi: J. Soc. Mater. Sci. Jpn., 34 (1985), 648.

2) P.-S. Chen and R. C. Wilcox: Mater. Charact., 26 (1991), 9.

3) P. Werth and S. Scherer: Proc. of 15th Int. Conf. on Pattern Recognition (ICPR 2000), Vol. 3, ICPR2000 Committee, Barcelona, (2000), 738.

4) J. Jun and S. Sakai: J. Soc. Mater. Sci. Jpn., 50 (2001), 1176.

5) T. Kobayashi and D. A. Shockey: Metall. Trans., 18A (1987), 1941.

6) J. Stampfl, S. Scherer, M. Berchthaler, M. Gruber and O. Kolednik: Int. J. Fract., 78 (1996), 35

7) J. Stampfl and O. Kolednik: Int. J. Fract., 101 (2000), 321.

8) Y. Kimura and M. Tanaka: Proc. of the 4th Int. Conf. on Materials for Resources, Vol. 2, Programming Committee of ICMR2001, Akita, (2001), 249.

9) B. B. Mandelbrot, D. E. Passoja and A. J. Paullay: Nature, 308 (1984), 721

10) R. H. Dauskardt, F. Haubensak and R. O. Ritchie: Acta Metall., 38 (1990), 142.

11) M. Tanaka: J. Mater. Sci., 27 (1992), 4717.

12) A. M. Gokhale, W. J. Drury and F. Mishra: ASTM STP1203, ed. by J. E. Masters and L. N. Gilbertson, American Society for Testing and
Materials, Philadelphia, PA, (1993), 3.

13) V. Y. Milman, N. A. Stelmashenko and R. Blumenfeld: Prog. Mater. Sci., 38 (1994), 425.

14) X. W. Li, J. F. Tian Y. Kang and Z. G. Wang: Scr. Metall. Mater., 33 (1995), 803.

15) M. Tanaka, A. Kayama and R. Kato: J. Mater. Sci. Lett., 18 (1999), 107.

16) E. Hornbogen: Z. Metallkd., 78 (1987), 622.

17) M. Tanaka and H. Iizuka: Z. Metallkd., 82 (1991), 442.

18) T. Nishihara: J. Jpn. Inst. Met., 57 (1993), 209.

19) P. Streitenberger, D. Förster, G. Kolbe and P. Veit: Scr. Metall. Mater., 33 (1995), 541.

20) B. B. Mandelbrot: The Fractal Geometry of Nature, transl. by $\mathrm{H}$. Hironaka, Nikkei Science, Tokyo, (1985).

21) H. Takayasu: Fractals in the Physical Sciences, Manchester University Press, Manchester and New York, (1990), 11

22) Y. H. Sun and H. Sunada: J. Soc. Mater. Sci. Jpn., 50 (2001), 1181

23) T. Kuroda, K. Ikeuchi, K. Nakade and Y. Matsuda: J. Soc. Mater. Sci. Jpn., 50 (2001), 1170.

24) M. Tanaka, A. Kayama, Y. Sato and Y. Ito: J. Mater. Sci. Lett., 17 (1998), 1715.

25) A. Kayama, M. Tanaka and R. Kato: J. Mater. Sci. Lett., 19 (2000), 565.

26) D. Hull: Fractography, Cambridge University Press, Cambridge, (1999).

27) M. Tanaka: Z. Metallkd., 84 (1993), 697. 